\title{
Ontic structural realism and the interpretation of quantum mechanics
}

\author{
Michael Esfeld
}

Received: 19 October 2011 / Accepted: 12 April 2012 / Published online: 9 May 2012

(C) Springer Science+Business Media B.V. 2012

\begin{abstract}
This paper argues that ontic structural realism (OSR) faces a dilemma: either it remains on the general level of realism with respect to the structure of a given theory, but then it is, like epistemic structural realism, only a partial realism; or it is a complete realism, but then it has to answer the question how the structure of a given theory is implemented, instantiated or realized and thus has to argue for a particular interpretation of the theory in question. This claim is illustrated by examining how OSR fares with respect to the three main candidates for an ontology of quantum mechanics, namely many worlds-type interpretations, collapse-type interpretations and hidden variable-type interpretations. The result is that OSR as such is not sufficient to answer the question of what the world is like if quantum mechanics is correct.
\end{abstract}

Keywords Ontic structural realism $\cdot$ Epistemic structural realism $\cdot$ Scientific realism · Quantum mechanics · Entanglement · Many worlds interpretation · Bohmian mechanics · GRW

\section{Introduction}

Structural realism in contemporary philosophy of physics can be traced back to Worrall (1989). According to the position that Worrall introduces as epistemic structural realism (ESR), there is continuity in the mathematical structure of mature scientific theories through theory change. This continuity justifies adopting a realist attitude as regards structure in the sense that the mathematical structure of a mature scientific theory reveals the way in which the entities in the domain of the theory in question are related with each other. ESR hence is a partial realism: it is a realism about structure, but it maintains that we do not know the nature or the essence of the

\footnotetext{
M. Esfeld $(\bowtie)$

Department of Philosophy, University of Lausanne, Lausanne, Switzerland e-mail: Michael-Andreas.Esfeld@unil.ch
} 
entities that implement, instantiate or realize the structure in question. This view provokes the objection that it is not possible to draw within a scientific theory a meaningful distinction between claims about the structure of the entities in its domain and claims about what these entities are, their nature (Psillos 1999, ch. 7). Ontic structural realism (OSR), introduced by Ladyman (1998), seeks to avoid this objection by maintaining that all there is in the world is structure. OSR thus is intended to be a complete realism: it tells what makes up the world, namely certain structures, and it holds that we can in principle know all that there is in the world.

Maintaining that all there is in the world is structure raises the question what is meant by this claim, since the physical world obviously is not mathematical structure. But there is a clear and straightforward answer to this question that covers all variants of OSR that have been developed subsequent to Ladyman (1998): a physical structure is a network of physical relations whose instantiation does not require underlying objects that possess an intrinsic identity - that is, an identity which is independent of these relations, being constituted by intrinsic properties or a primitive thisness (haecceity). This is a general claim, but it has a substantial content: it rules out certain accounts of what the world is like, such as, for instance, Lewis' metaphysics of Humean supervenience (Lewis 1986, introduction).

The thesis of this paper is that the move from ESR to OSR is not sufficient to turn structural realism into a complete scientific realism: the claim that structure is all there is in the world and that there are no objects with (unknowable) intrinsic identities underlying structure leaves open how the structure in question is implemented, instantiated or realized. The proponents of OSR hence face a dilemma: either they remain on the general level of a commitment only to structure, but then their position merely is a partial realism, not substantially going beyond ESR; or they put forward a complete realism, but then they have to leave behind a realism that merely concerns the structure of a theory, specifying how the structure at issue is instantiated. In other words, they then have to engage in the controversies about the interpretation of the theory in question and argue for a particular interpretation.

Two clarifications are appropriate to avoid misunderstanding this thesis: (a) OSR moves beyond Worrall's ESR in that it abandons the commitment to unknowable intrinsic properties that constitute the identity of physical objects underlying structure. But doing away with such intrinsic properties is not sufficient to get from a partial to a complete realism: it leaves open how the structure in question is implemented in the physical world. There is a wider reading of ESR possible than Worrall's original one; according to this wider reading, ESR is a partial realism because it refuses to make a commitment as to how the structure in question is implemented (cf. the "liberal ESR" of Slowik 2012, sect. 1.1). (b) I deliberately use the rather vague phrase of leaving open how the structure that a given mature scientific theory poses is implemented, instantiated or realized in the physical world in order to avoid the objection of begging the question against OSR: if I talked in terms of leaving open what the objects (or the entities) are that implement the structure in question, one could retort that the point of OSR is to be committed to structures instead of objects. However, even if the commitment is supposed to be one to structures without objects, for the resulting view to be a complete realism, we have to be told how the structures in question are instantiated. 
In the following, I shall illustrate this thesis by considering non-relativistic quantum mechanics (QM), since QM is the physical theory that motivates the move from ESR to OSR in Ladyman (1998) and French and Ladyman (2003). However, it is important for the thesis of this paper that it is not limited to OSR as applied to QM. It concerns also OSR with respect to the other main fundamental physical theory, namely general relativity, as I shall point out at the end of the next section. In section 2 , I elaborate on the mentioned dilemma by assessing the arguments that proponents of OSR put forward on the basis of QM. This section is rather negative, its aim being to show the limits of these arguments. Section 3 then brings OSR together with the main interpretations of QM, evaluating to what extent OSR can serve as a guideline in the interpretation of QM. The paper ends with a brief assessment of the contribution that OSR makes for an ontology of QM.

\section{A dilemma for OSR}

Ladyman (1998) and French and Ladyman (2003) motivate the transition from ESR to OSR by drawing on the interpretation of QM. According to them, there is an underdetermination between an ontology of quantum objects as individuals and an ontology of quantum objects as non-individuals. That underdetermination is a challenge to realism. In order to meet that challenge, they propose to abandon the commitment to objects and to subscribe instead to an ontology of structures. The underdetermination that French and Ladyman have in view arises from the fact that they consider it to be a serious option to regard quantum objects as possessing a primitive thisness (haecceity) so that permuting these objects amounts to a real difference. However, attributing a primitive thisness to objects is a purely metaphysical move that one can always make, physics be as it may. As regards the physics, there is no underdetermination arising from the commitment to a primitive thisness being a physical option (cf. already the criticism of Cao 2003, pp. 62-64).

Nonetheless, one may maintain that there is an underdetermination between an ontology of quantum objects as individuals and an ontology of quantum objects as non-individuals. Consider as one example the EPR-Bohm experiment on two quantum systems of spin $1 / 2$ (or two photons) in the singlet state so that the possible values of spin in any direction of the two systems are correlated whatever their spatiotemporal distance is (Einstein et al. 1935; Bohm 1951, pp. 611-622). Bell's theorem (1964, reprinted in Bell 1987, ch. 2) proves that it is not possible to account for these correlations on the assumption that the preparation of the singlet state at the source of the experiment is the common cause of them. Nonetheless, it is in principle possible to construct common cause models that explain these correlations (e.g. Suárez 2007; San Pedro 2012), or to assume backwards causation (e.g. Price 1996, ch. 8 and 9) or superluminal signals that connect the two wings of an EPR-Bohm experiment (e.g. Chang and Cartwright 1993, part III). In all these models, quantum systems are taken to be individual particles with a well-defined identity in time constituted by physical properties that are hidden variables (for they are not taken into account in standard $\mathrm{QM}$ ); but there is no question in these models of these systems possessing a primitive thisness. 
As regards the option to consider quantum systems as non-individuals, the idea is that there is a numerical plurality of quantum objects, but that these objects neither have an identity at a time that distinguishes each of them from all the other ones, nor do they have an intertemporal identity. Thus, there is no fact of the matter which one of the two quantum objects prepared in the singlet state at the source of an EPRBohm experiment is later measured in the left wing and which one is measured in the right wing of the experiment. Consequently, one may speak of an underdetermination on the basis of the experimental data between an ontology of quantum systems as individuals and an ontology of quantum systems as non-individuals.

How does OSR dissolve this underdetermination? If OSR is conceived as an ontology of structures by contrast to an ontology of objects, eliminating the commitment to objects in the domain of fundamental physics, three forceful objections apply: (a) the metaphysical objection that relations require relata as that what stands in the relations, even if the relata do not have to possess an intrinsic identity (e.g. Cao 2003; Psillos 2006); (b) the logical objection that in formulating a physical theory in set theoretic terms, one quantifies over objects (but see Bain 2012 who pleads in favour of using category theory instead of set theory to avoid this objection); (c) the physical objection that QM talks in terms of countable quantum systems and thereby is committed to objects (Ainsworth 2010, p. 53).

Reacting to these objections, French and Ladyman can be read as having in the further development of OSR moved away from an eliminativism about objects to a position according to which objects are constituted by relations (Ladyman and Ross 2007, ch. 2 to 4, French 2010). Furthermore, other versions of OSR have been set out that explicitly admit objects, reaching to a moderate OSR that acknowledges objects on the same ontological footing as relations (Esfeld 2004; Esfeld and Lam 2008; Floridi 2008) (see Ainsworth 2010 for an overview). Recent publications suggest a convergence of these different versions of OSR in the following sense: OSR acknowledges what French (2010, sect. 7) dubs "thin objects", namely objects for which it is constitutive to stand in the relations (the structure) in question. Esfeld and Lam (2011, sect. 8.3) propose to regard the relations (structures) as the ways (modes) in which the objects are. In short, the objects that OSR admits do not have a being in distinction to the relations (structures) in which they stand. It then seems to be merely a verbal matter whether or not one grants the label "objects" to entities that stand in relations without possessing an identity independently of these relations.

Be that as it may, the important point for present purposes is that OSR is not a tertium quid that leaves the underdetermination between the view of quantum systems as individuals and the view of non-individuals behind, but an elaboration on what objects as non-individuals can be, namely objects for which it is constitutive to stand in certain relations. However, a new form of underdetermination now arises: saying that QM is committed to certain structures, because it is constitutive for the objects over which QM quantifies to stand in certain relations, is not sufficient as an ontology for QM, since it does, for instance, nothing to account for the measured correlations in the EPR-Bohm experiments. If one considers the proposals that set out to explain these correlations, then some of them indeed regard quantum objects as non-individuals. Nonetheless, they account for these correlations not through remaining on the level of taking quantum objects to be non-individuals, but by advancing concrete proposals as to what these objects are and how their states develop in time. I 
shall elaborate on this issue in the next section. Let us note here simply that the arguments about quantum objects being non-individuals are not sufficient to establish OSR as a complete realism in the domain of QM.

In the context of considering quantum systems as non-individuals in the sense that there is a numerical plurality of them, but they neither possess an identity at a time that distinguishes each of them from all the other ones nor an intertemporal identity, it is also possible to ground OSR with respect to QM on an argument to the effect that quantum objects are only weakly discernible, being linked by irreflexive and symmetric relations that do not supervene on any intrinsic properties (see notably Saunders 2006 for that argument and Muller 2011 on the link between quantum objects being weakly discernible and OSR). However, it can be objected that this move amounts to grounding OSR in certain group-theoretical symmetry considerations that provide abstract theoretical descriptions of the properties in the domain under consideration, but that do not seize the concrete property tokens themselves (Morganti 2011, sect. 4). In other words, the possibility of a description of a given domain in terms of certain symmetries does not imply that it is constitutive for the objects in that domain to stand in certain concrete physical relations. The argument from symmetry considerations therefore also fails to establish OSR as a complete realism.

However, there is a possibility to build an argument for OSR with respect to QM directly on certain concrete physical relations that is not touched by this objection. Entanglement is the physical basis of the view that QM is committed to certain structures instead of objects with an intrinsic identity. Thus, for instance, in the EPRBohm experiment, the two quantum systems are in an entangled spin state instead of having a well-defined state each. One can directly base OSR on considering entanglement to be the distinctive feature of the quantum domain and spell out entanglement in terms of the non-separability of the states of quantum systems (Esfeld 2004). Nonetheless, the above mentioned objections apply: according to common cause models, backward causation models and models in terms of superluminal signals, entanglement is an epistemic instead of an ontological feature, concerning only our knowledge of quantum systems; these systems themselves are individuals that always have a well-defined state each.

Furthermore, and more importantly for the purposes of this paper, the assumption of non-separability alone (structure of entangled quantum systems) does not amount to an explanation of the non-local correlations, which are, for instance, measured in the EPR-Bohm experiments: it does not answer the question why and how a local interaction in one wing of such an experiment has consequences for the probabilities for measurement outcomes consisting in a change in a macroscopic device in the other, space-like separated wing of the experiment. One may say that the obvious reason for this lack is that OSR puts forward an ontology by telling us what there is in the world, namely certain physical structures, but not a dynamics - that is, it remains silent on the question of how the structures to which it is committed develop in time.

The main proponents of OSR have always conceived physical structures as modal and thus in a non-Humean way (French and Ladyman 2003; Ladyman and Ross 2007, ch. 2 to 4, French 2010, sect. 3; but see Lyre 2010 and 2012 for a dissenting voice). There is a clear reason for doing so: the identity of a structure consists in its playing a certain nomological role. By contrast, according to the position known 
today as Humean metaphysics, properties play a nomological role only contingently (see in particular Lewis 2009). Thus, in Humean metaphysics, one and the same qualitative, intrinsic property can play the charge role in one world and the mass role in another world. However, it does not make sense to conceive one and the same structure playing in one world, say, the role of the quantum structures of entanglement and in another world the role of the metrical-gravitational structures. In short, if there are structures, then it is essential for a structure to occupy a certain nomological role.

Recently, it has been proposed to regard the nomological role that structures necessarily play as a causal role so that the structures to which OSR is committed are causally efficacious in themselves, being dispositions or powers (Esfeld 2009; see Psillos 2012 for general objections against this proposal). In short, the idea is to include a dynamics in OSR by conceiving the structures as generating a dynamics through their being causally efficacious. However, this move is not sufficient to answer the above-mentioned question: the structures of quantum entanglement are causally efficacious in that they bring about the experimental results, such as, for instance, the measured correlations in an EPR-Bohm experiment. But how do they do so? Do they do so through decoherence and a branching of the universe? Do they do so through state reductions? Or do they do so in yet another way? Again, the mentioned dilemma is obvious: either OSR remains on a general level of posing structures - and conceiving them as modal or even causal does not change anything as far as the issue of remaining on a general level is concerned -, but then it is, like ESR, only a partial realism, remaining silent on the question of how the structures are implemented and how the way in which they are implemented brings about the experimental results; or it is a complete realism, but then it has to go beyond the general commitment to structures, answering the question how precisely the structures to which QM is committed are implemented in the physical domain.

The central problem therefore is not that OSR lacks a dynamics, but that OSR provides only a general scheme for an ontology of the physical domain, being itself not sufficient to spell out such an ontology. One may reply that this deficiency concerns only OSR about QM and is due to the fact that QM runs into the famous measurement problem. In other words, this deficiency does not touch OSR as such, but is a consequence of the fact that the formalism or structure of QM, by contrast to the formalism or structure of other physical theories, raises a particular problem of interpretation.

OSR has also been proposed with respect to the other current fundamental physical theory, namely general relativity (see notably Esfeld and Lam 2008). In this case, it is maintained that the metrical properties are relational instead of intrinsic properties so that general relativity theory is committed to a structure of metrical relations. It has been claimed that OSR is a tertium quid as regards the traditional opposition between substantivalism and relationalism about space-time (Dorato 2000, 2008). But there is no reason for such a claim. OSR with respect to space-time tells us that there are metrical structures; however, it remains silent on the issue of how these structures are implemented. If one maintains that these structures are implemented by relations among space-time points, then OSR is a version of sophisticated substantivalism (e.g. Hoefer 1996) or metrical essentialism (e.g. Maudlin 1989; Bartels 1996). Roughly speaking, these positions maintain that there are space-time points; however, these are 
not manifold points, but the metrical properties are constitutive of their identity. OSR then adds the claim that the metrical properties are relational instead of intrinsic properties. If, by contrast, one maintains that the metrical properties are instantiated by material entities, then OSR about space-time is a version of relationalism. In a recent paper, Slowik (2012, sect. 1) elaborates on this underdetermination problem for OSR about space-time and challenges on this basis the claim that OSR is superior to ESR as regards a scientific realist position.

In sum, OSR with respect to general relativity theory faces the same dilemma as OSR with respect to QM: either OSR remains on a general level of posing metrical structures, but then it is, like ESR, only a partial realism, remaining silent on the question of how these structures are implemented in the physical domain; or it is a complete realism, but then it has to go beyond the general commitment to metrical structures, answering the question how the metrical structures are implemented. This dilemma concerns OSR in general, to whatever physical theory it is applied. The point at issue is not that there have to be objects (or, in general, some entities or other) underlying the structures in question, but that a realist position has to spell out in concrete terms how the structures to which a certain theory is committed are implemented, instantiated or realized in the physical domain. ${ }^{1}$

\section{OSR applied to the main interpretations of QM}

The preceding section resulted in the negative claim that the arguments for OSR from quantum physics fail to establish OSR as a full-blown realist position in the philosophy of QM: these arguments are not capable to turn OSR into a complete realist position by contrast to the partial realism that ESR yields. But one can formulate this result also in positive terms: OSR is a partial realism with respect to QM in that it provides a framework for an ontology to which QM is committed under any reasonable interpretation, namely an ontology according to which there are certain structures (i.e. structures of entanglement) in the domain of quantum physics instead of objects with an intrinsic identity. If one sets out to develop this partial realism into a complete realism, then one has to settle for a particular interpretation of QM, spelling out how the structures in question are implemented and what their dynamics is. In other words, the idea is that the appropriate place for OSR in the interpretation of QM is to serve as a guideline for an ontology for QM instead of itself providing a complete ontology. To give this idea a fair hearing, let us leave aside the abovementioned models that seek to account for the experimentally proven quantum nonlocality in terms of hidden common causes, backwards causation or superluminal causation; it is obvious that these models do not fall within OSR, and one can with reason claim that none of them has been worked out in a physically precise manner hitherto. Let us assess whether OSR can serve as a guideline for an ontology for QM in bringing out a meaningful commitment to structures that is common to the three main types of interpretations of QM.

\footnotetext{
${ }^{1}$ In my earlier publications in favour of OSR (Esfeld 2004 and 2009; Esfeld and Lam 2008 and 2011), I failed to see this point. The present paper corrects my earlier publications in arguing that we have to go beyond OSR in order to achieve a staisfactory scientific realist position in the philosophy of physics.
} 
To introduce these interpretations, consider the above-mentioned measurement problem, which is indeed the central problem in the interpretation of QM. Maudlin (1995, p. 7) provides the following precise formulation of this problem:

1.A The wave-function of a system is complete, i.e. the wave-function specifies (directly or indirectly) all of the physical properties of a system.

1.B The wave-function always evolves in accord with a linear dynamical equation (e.g. the Schrödinger equation).

1.C Measurements of, e.g., the spin of an electron always (or at least usually) have determinate outcomes, i.e., at the end of the measurement the measuring device is either in a state which indicates spin up (and not down) or spin down (and not up).

Since the conjunction of these three propositions is inconsistent, we have to give up at least one of them and thus get to three main types of interpretation of QM. In brief, many worlds-type interpretations give up 1C, collapse-type interpretations give up $1 \mathrm{~B}$ and hidden variable-type interpretations give up $1 \mathrm{~A}$.

If one rejects $1 \mathrm{C}$, one has to replace $1 \mathrm{C}$ with an account of how it comes about that it seems to observers that there are determinate values of properties of themselves as well as their environment. In order to achieve such an account, it is common to draw on decoherence. In brief, the idea is that the physical significance of the vanishing of the interference terms between the superposed correlations known as decoherence is that it induces a splitting of the universe into many non-interfering branches such that each of the superposed correlations constitutes at least one branch of the universe. Thus, there is one branch in which the electron has spin up, the measuring device indicates spin up and the observer is conscious of the measuring device indicating spin up; and there is another branch in which the same electron has spin down, the same measuring device indicates spin down and the same observer is conscious of the measuring device indicating spin down. Since there are many measurements for which there are infinitely many possible outcomes - position measurements are a case in point -, this view is committed to maintaining that decoherence leads to the emergence of infinitely many branches. This position is therefore known as the many worlds interpretation of QM, going back to Everett (1957) (see Wallace 2010 for a concise statement and in general the papers in Saunders et al. 2010 for discussion).

On the one hand, this interpretation can be seen as illustrating OSR: there is a structure being implemented by ("thin") objects that are copied many times in noninterfering branches of the universe and whose dynamical properties have determinate values that are correlated within each branch ("relative state"). There is no intrinsic identity of objects in a branch, since the values of their properties depend on the values of the properties of the other objects in that branch. That structure develops according to a global law applying to the universe as a whole (such as the Schrödinger equation in QM). On the other hand, one can maintain that the central characteristic feature of the many worlds interpretation is not its commitment to certain structures as brought out by OSR, but its claim that there are many noninterfering branches of the universe.

The standard textbooks from von Neumann (1932) on present a formulation of QM that rejects 1B: the wave-function completely describes the properties of physical systems, but under some circumstances - measurements being a case in point - 
quantum systems change in such a way that they acquire a determinate value of dynamical properties, that change being represented by the collapse of the wavefunction. The only proposal that makes this idea precise goes back to Ghirardi et al. (1986) (GRW). GRW add a stochastic term to the Schrödinger equation such that, in brief, a single microscopic quantum system has a very low objective probability to undergo a spontaneous localization. However, when it comes to a macroscopic system that is composed of a huge number of microscopic quantum systems, one of these microscopic systems will immediately undergo a spontaneous localization so that, due to the entanglement, the whole system will be localized.

Nonetheless, it remains to be spelled out what exactly in the physical world the GRW dynamics represents - in other words, what the ontology of GRW is. If one takes textbook QM literally, the view is suggested that a quantum system such as an electron, when not having a determinate value of position, is smeared out in space. This is indeed the reading of the physical significance of GRW that Ghirardi himself favours in proposing a mass density ontology (Ghirardi et al. 1995): the mass of, say, an electron when it has not a determinate position is literally smeared out in physical space, creating thus a mass density distribution.

On the one hand, again, the GRW mass density ontology can be seen as illustrating OSR: there is a structure of correlated values of properties, which are smeared out values implemented by a mass density (density of stuff in physical space), instead of properties that provide for an intrinsic identity of the entities that instantiate them. This structure develops as a whole according to a certain law of motion, which in the case of GRW attributes to the structure a disposition or propensity for spontaneous localization whose strength depends on the number of elementary quantum systems involved in the structure in question (see Dorato and Esfeld 2010 for dispositions in GRW). On the other hand, again, one can maintain that the central characteristic feature of the GRW mass density ontology is not its commitment to certain structures as brought out by OSR, but its claim that quantum systems are literally smeared out in physical space, thus constituting a mass density distribution, which contracts spontaneously under certain circumstances.

There is another ontology for the GRW dynamics proposed in the literature that entirely abandons the idea of there being smeared out values in the physical world. That ontology is due to Bell (1987, p. 205). According to what is today known as the GRW flash theory (this term goes back to Tumulka 2006), quantum systems have a determinate value of position only when the wave-function as developing according to the GRW equation indicates such a value - that is, when a spontaneous localization occurs -, and these sparse determinate positions are all there is in space-time. In other words, the spontaneous localizations that GRW postulate are conceived as flashes centred around space-time points, and these flashes are all there is. Starting with an initial distribution of flashes, the wave-function determines the probabilities for the occurrence of further flashes (see Allori et al. 2008 for an illuminating comparison of the ontologies of GRW mass density, GRW flash and Bohmian mechanics).

On the one hand, again, the GRW flash ontology can be seen as illustrating OSR: the universe is a structure of correlated flashes. That structure exists in fourdimensional space-time, the flashes being events occurring at space-time points. By contrast to the structure of the GRW mass density, the flash structure is not 
continuous in space-time, since the flashes are sparsely distributed. That structure develops as a whole according to a certain law of motion, which in the case of the GRW flash ontology attributes to any given structure of flashes a disposition or propensity to bring about further correlated flashes. On the other hand, again, one can maintain that the central characteristic feature of the GRW flash ontology is not that it is committed to correlated flashes (structures of flashes), but that it poses only flashes as that what exists in space-time.

Interpretations of QM that reject proposition 1A above are known as hidden variable theories. As already indicated, hidden variable models that refuse to grant any ontological significance to quantum entanglement, seeking to account for the experimentally proven quantum non-locality in terms of hidden common causes, backwards causation or superluminal signals fall outside the scope of OSR; but none of these models amounts to a main contender in the interpretation of QM. The decisive question for assessing the claim that OSR can serve as a guideline for an ontology for QM is how it fares with respect to the only precisely worked out hidden variable theory, namely Bohm's quantum theory, going back to Bohm (1952) (to be correct, this is not a hidden variable theory, since it is odd to describe position as a "hidden variable").

It would be unfair to dismiss this question by pointing out that Bohm's theory is not Lorentz-invariant. Collapse-type interpretations face the same difficulty (although Tumulka 2006 makes a significant progress in this respect for the GRW flash ontology). Furthermore, depending on how the branching of the universe is conceived, it is not evident how many-worlds type interpretations can be Lorentzinvariant either (cf. Barrett 1999, pp. 159-160). In general, the experimentally proven quantum non-locality as exhibited in the EPR-Bohm experiment carries over to quantum field theory: the probabilities for measurement outcomes at a certain space-time point or region depend on what there is at points or regions that are separated by a space-like interval from that point. As regards the demand for a Lorentz-invariant account of these correlations, quantum field theory is in no better position than non-relativistic QM.

In enquiring whether OSR can be applied to Bohm's theory, let us focus on the contemporary conceptualization of this theory known as Bohmian mechanics, which abandons the quasi-Newtonian formulation of the theory in terms of a quantum potential (see the papers collected in Dürr et al. 2012 as well as Belot 2012 for a recent philosophical discussion). According to Bohmian mechanics, what there is in the quantum domain are particles that always have a definite position and thus a welldefined trajectory in space-time. These particles move according to a linear and deterministic law of motion. That law makes the temporal development of the position of any particle (i.e. its velocity) dependent on the positions of all the other particles. Bohmian particles hence distinguish themselves by their position (no two particles can have the same value of position at a given time); they are absolutely discernible objects. However, this fact does not hinder applying OSR to Bohmian mechanics: OSR does not entail a failure of discernibility (see Ainsworth 2011).

Bohmian particles can be seen as implementing a structure in the sense of OSR because of the holistic dynamics to which they are subject: the temporal development of the position of any particle is correlated with the development of the positions of 
all the other particles in the universe. If one takes the Bohmian law of motion and the quantum mechanical wave-functions that figures in this law as referring to a property of the particles, this is a holistic and thus non-separable property, namely a disposition for a certain form of motion of the particles (see Belot 2012, pp. 77-80). In other words, it is a structure that takes all the particles as relata. In short, on Bohmian mechanics, there is a structure of correlated particle positions. That structure develops as a whole according to a certain law of motion. In sum, again, on the one hand, Bohmian mechanics can be considered as illustrating OSR as well; but on the other hand, again, one can maintain that the central characteristic feature of Bohmian mechanics is not that it is committed to structures implemented by correlated particle positions, but that it poses particle positions as the basic ontology for QM.

\section{Conclusion}

OSR is not an interpretation of QM in addition to many worlds-type interpretations, collapse-type interpretations, or hidden variable-type interpretations. As the discussion of the arguments for OSR from QM in section 2 above has shown, OSR is not in the position to provide on its own an ontology for QM, since it does not reply to the question of what implements the structures that it poses. Nonetheless, there is a positive contribution that OSR can make to the search for an ontology for QM, namely to bring out an ontological commitment that applies to QM under any well-grounded interpretation, that is, the commitment to there being a certain sort of structures in the domain of quantum physics instead of objects with an intrinsic identity. A similar remark applies to OSR with respect to any other scientific theory (cf. the remarks at the end of section 2 about OSR applied to general relativity theory).

But is it true that OSR provides a general and substantial framework that any detailed reasonable interpretation of QM has to respect, that framework consisting in the claim that there are certain structures in the domain of quantum physics by contrast to objects with an intrinsic identity? Infinitely many branches of the universe with correlated values of properties in each branch ("relative states"), density of stuff or mass in four-dimensional space-time (smeared-out values), pointlike flashes sparsely distributed in space-time and particles with definite trajectories in space-time are radically different proposals for an ontology of QM, although all these ontologies can be regarded as being committed to certain structures, namely structures of entanglement. However, these ontologies are radically different not because they pose different sorts of objects in addition to agreeing on being committed to structures of entanglement, but because they spell out what entanglement means in radically different ways: superpositions of correlations in the form of objects being many times copied across different branches of the universe whose properties are correlated within each branch; superpositions of correlations in the form of overlapping smeared-out values in physical space making up a continuous distribution of stuff (mass density) in space; correlated flashes occuring at space-time points; position values of particles that develop in time in a correlated manner. 
For the full-blown scientific realist, what counts is to work out and assess these different ontologies. The partial realist, by contrast, may stay with the claim that these different ontologies exhibit a commitment to a common, albeit general structure. However, in order to vindicate that latter claim, the move from ESR to OSR seems not necessary. To put it in a nutshell, given the radical differences in the precise account of what entanglement means among the proposals for an ontology for QM that can all be seen as illustrating OSR, one may wonder whether OSR is suitable as a guideline for an ontology for QM that tells us substantially more than what is given by the experimental and mathematical constraints for an ontology for QM, namely in particular the experimentally proven quantum non-locality and its mathematical analysis in Bell's theorem, showing that no local hidden variable ontology is available for QM.

In conclusion, after more than a decade of elaboration and debate on OSR about QM, it seems that the impact that OSR can have on providing an answer to the question of what the world is like if QM is correct is rather limited. From a scientific realist perspective, the crucial issue is the assessment of the pros and cons of the various detailed proposals for an ontology of QM, as it was before the appearance of OSR on the scene.

\section{References}

Ainsworth, P. M. (2010). What is ontic structural realism? Studies in History and Philosophy of Modern Physics, 41, 50-57.

Ainsworth, P. M. (2011). Ontic structural realism and the principle of the identity of indiscernibles. Erkenntnis, 75, 67-84.

Allori, V., Goldstein, S., Tumulka, R., \& Zanghi, N. (2008). On the common structure of Bohmian mechanics and the Ghirardi-Rimini-Weber theory. British Journal for the Philosophy of Science, 59, $353-389$.

Bain, J. (2012). Category-theoretic structure and radical ontic structural realism. Forthcoming in Synthese. doi:10.1007/s11229-011-9896-6.

Barrett, J. A. (1999). The quantum mechanics of minds and worlds. Oxford: Oxford University Press.

Bartels, A. (1996). Modern essentialism and the problem of individuation of spacetime points. Erkenntnis, $45,25-43$.

Bell, J. S. (1987). Speakable and unspeakable in quantum mechanics. Cambridge: Cambridge University Press.

Belot, G. (2012). Quantum states for primitive ontologists. A case study. European Journal for Philosophy of Science, 2, 67-83.

Bohm, D. (1951). Quantum theory. Englewood Cliffs: Prentice-Hall.

Bohm, D. (1952). A suggested interpretation of the quantum theory in terms of 'hidden' variables. Physical Review, 85, 166-193.

Cao, Tian Yu. (2003). Can we dissolve physical entities into mathematical structure? Synthese, 136, 57-71.

Chang, H., \& Cartwright, N. (1993). Causality and realism in the EPR experiment. Erkenntnis, 38, 169190.

Dorato, M. (2000). Substantivalism, relationism, and structural spacetime realism. Foundations of Physics, $30,1605-1628$.

Dorato, M. (2008). Is structural spacetime realism relationism in disguise? The supererogatory nature of the substantivalism/relationism debate. In D. Dieks (Ed.), The ontology of spacetime II (pp. 17-37). Amsterdam: Elsevier.

Dorato, M., \& Esfeld, M. (2010). GRW as an ontology of dispositions. Studies in History and Philosophy of Modern Physics, 41, 41-49.

Dürr, D., Goldstein, S., \& Zanghì, N. (2012). Quantum physics without quantum philosophy. Berlin: Springer. 
Einstein, A., Podolsky, B., \& Rosen, N. (1935). Can quantum-mechanical description of physical reality be considered complete? Physical Review, 47, 777-780.

Esfeld, M. (2004). Quantum entanglement and a metaphysics of relations. Studies in History and Philosophy of Modern Physics, 35, 601-617.

Esfeld, M. (2009). The modal nature of structures in ontic structural realism. International Studies in the Philosophy of Science, 23, 179-194.

Esfeld, M., \& Lam, V. (2008). Moderate structural realism about space-time. Synthese, 160, $27-46$.

Esfeld, M., \& Lam, V. (2011). Ontic structural realism as a metaphysics of objects. In A. Bokulich \& P. Bokulich (Eds.), Scientific structuralism (pp. 143-159). Dordrecht: Springer.

Everett, H. (1957). Relative state' formulation of quantum mechanics. Reviews of Modern Physics, 29, 454-462. Reprinted in B. S. DeWitt and N. Graham (eds.) (1973): The many-worlds interpretation of quantum mechanics. Princeton: Princeton University Press. pp. 141-149

Floridi, L. (2008). A defence of informational structural realism. Synthese, 161, 219-253.

French, S. (2010). The interdependence of structure, objects and dependence. Synthese, 175, 177-197.

French, S., \& Ladyman, J. (2003). Remodelling structural realism: quantum physics and the metaphysics of structure. Synthese, 136, 31-56.

Ghirardi, G. C., Grassi, R., \& Benatti, F. (1995). Describing the macroscopic world: closing the circle within the dynamical reduction program. Foundations of Physics, 25, 5-38.

Ghirardi, G. C., Rimini, A., \& Weber, T. (1986). Unified dynamics for microscopic and macroscopic systems. Physical Review D, 34, 470-491.

Hoefer, C. (1996). The metaphysics of space-time substantivalism. Journal of Philosophy, 93, 5-27.

Ladyman, J. (1998). What is structural realism? Studies in History and Philosophy of Modern Science, 29, $409-424$.

Ladyman, J., \& Ross, D. (2007). Every thing must go. Metaphysics naturalised. Oxford: Oxford University Press.

Lewis, D. (1986). Philosophical papers. Volume 2. Oxford: Oxford University Press.

Lewis, D. (2009). Ramseyan humility. In D. Braddon-Mitchell \& R. Nola (Eds.), Conceptual analysis and philosophical naturalism (pp. 203-222). Cambridge (Massachusetts): MIT Press.

Lyre, H. (2010). Humean perspectives on structural realism. In F. Stadler (Ed.), The present situation in the philosophy of science (pp. 381-397). Dordrecht: Springer.

Lyre, H. (2012). Structural invariants, structural kinds, structural laws. In D. Dieks, W. J. Gonzalez, S. Hartmann, M. Stöltzner, \& M. Weber (Eds.), Probabilities, laws, and structures (pp. 169-181). Dordrecht: Springer.

Maudlin, T. (1989). The essence of space-time. In A. Fine \& J. Leplin (Eds.), Proceedings of the 1988 Biennial Meeting of the Philosophy of Science Association. Volume 2 (pp. 82-91). East Lansing: Philosophy of Science Association.

Maudlin, T. (1995). Three measurement problems. Topoi, 14, 7-15.

Morganti, M. (2011). Is there a compelling argument for ontic structural realism? Philosophy of Science, $78,1165-1176$.

Muller, F. A. (2011). Withering away, weakly. Synthese, 180, 223-233.

Price, H. (1996). Time's arrow and Archimedes' point. New directions for the physics of time. Oxford: Oxford University Press.

Psillos, S. (1999). Scientific realism. How science tracks truth. London: Routledge.

Psillos, S. (2006). The structure, the whole structure and nothing but the structure? Philosophy of Science, $73,560-570$.

Psillos, S. (2012). Adding modality to ontic structuralism: an exploration and critique. In E. Landry \& D. Rickles (Eds.), Structural realism. Structure, object, and causality (pp. 169-185). Dordrecht: Springer.

San Pedro, I. (2012). Causation, measurement relevance and no-conspiracy in EPR. European Journal for Philosophy of Science, 2, 137-156.

Saunders, S. (2006). Are quantum particles objects? Analysis, 66, 52-63.

Saunders, S., Barrett, J., Kent, A., \& Wallace, D. (Eds.). (2010). Many worlds? Everett, quantum theory, and reality. Oxford: Oxford University Press.

Slowik, E. (2012). On structuralism's multiple paths through spacetime theories. European Journal for Philosophy of Science, 2, 45-66.

Suárez, M. (2007). Causal inference in quantum mechanics: a reassessment. In F. Russo \& J. Williamson (Eds.), Causality and probability in the sciences. London: London College.

Tumulka, R. (2006). A relativistic version of the Ghirardi-Rimini-Weber model. Journal of Statistical Physics, 125, 821-840. 
von Neumann, J. (1932). Mathematische Grundlagen der Quantenmechanik. Berlin: Springer. English translation: Mathematical foundations of quantum mechanics. Translated by R. T. Beyer. Princeton: Princeton University Press 1955.

Wallace, D. (2010). Decoherence and ontology (or: How I learned to stop worrying and love FAPP). In S. Saunders, J. Barrett, A. Kent, \& D. Wallace (Eds.), Many worlds? Everett, quantum theory, and reality (pp. 54-72). Oxford: Oxford University Press.

Worrall, J. (1989). Structural realism: the best of two worlds? Dialectica, 43, 99-124. Reprinted in D. Papineau (ed.) (1996): The philosophy of science. Oxford: Oxford University Press. pp. 139-165. 